\title{
Reducing Contrast-Agent Volume And Radiation Dose In CT With 90Kvp Tube Voltage, High Tube Current Modulation, And Advanced Iteration Algorithm
}

\section{Min Su Park}

Hallym University Sacred Heart Hospital

Hong II Ha ( $\nabla$ ha.hongil@gmail.com )

Hallym University Sacred Heart Hospital

Jhii-Hyun Ahn

Yonsei University Wonju College of Medicine, Wonju Severance Christian Hospital

\section{Sang Min Lee}

Hallym University Sacred Heart Hospital

In Jae Lee

Hallym University Sacred Heart Hospital

\section{Research Article}

Keywords: Tomography, X-ray Computed, contrast media, dose reduction, image enhancement, radiation dosage

Posted Date: March 3rd, 2022

DOI: https://doi.org/10.21203/rs.3.rs-1347201/v1

License: (c) (i) This work is licensed under a Creative Commons Attribution 4.0 International License.

Read Full License 


\section{Abstract}

The purpose of this study was to evaluate the image quality and the radiation dose in pancreatic dynamic CT with $90 \mathrm{kVp}$ tube voltage and reduction of the standard amount of contrast agent, compared with 100kVp PDCT. 51 patients who underwent both CT protocols were included in this retrospective study. One reviewer measured the attenuation values of the abdominal organs and image noise for objective image quality analysis. Two radiologists evaluated the five categories of image qualities for subjective image quality analysis. As a result, the total amount of the contrast agent, the radiation dose, and image noise decreased in the low-kVp group, by $24.4 \%, 31.7 \%$, and $20.6 \%$, respectively $(p<0.001)$. The intraobserver and interobserver agreements were moderate to substantial $(k=0.4-0.8)$. The SNR and CNR of the almost organs in the low-kVp group were significantly higher $(p<0.001)$. Except for lesion conspicuity, both reviewers judged that the subjective image quality of the low-kVp group was better ( $p<$ 0.001). With $90 \mathrm{kVp}$ tube voltage, reduced contrast agent volume with advanced iteration and high tube current modulation achieved radiation dose reduction of $31.7 \%$, as well as better image quality and diagnostic confidence.

\section{Introduction}

The rapidly increasing utilization of computed tomography (CT) has raised concerns regarding potential hazards due to CT radiation [1]. In conjunction with radiation hazards, CT technology has been developed to achieve an appropriate balance between image quality, reduction in radiation dose, and the amount of contrast material $[2,3]$. The following technological advances, such as a decrease in tube potential and an increase in compensatory tube current as well as iterative reconstruction (IR), have contributed the most to this $[4,5]$. Low-tube-voltage imaging causes increased image noise and susceptibility to beam hardening artefacts, simultaneously resulting in degradation of image quality [6]. This disadvantage of low-tube-voltage imaging is compensated by IR. IR not only improves the overall image quality by reducing image noise but also contributes to improved diagnostic confidence [6, 7]. Based on these advantages, low tube voltage and IR techniques have been widely used in CT scans in children, CT angiography, and chest CT [8-11].

However, low-tube-voltage imaging is limited in adult abdominal CT. A decrease in radiation penetration resulting from low tube voltage may lead to an increase in image noise and create a blotch image artefact in solid organs, such as the liver, resulting in image quality degradation [12, 13]. Low-tube-voltage imaging shows higher iodine contrast enhancement because the mean photon energy approaches the iodine kedge of $33 \mathrm{keV}[14,15]$. Higher iodine contrast enhancement is a double-edged sword in adult abdominal CT. This improves the contrast-noise ratio (CNR) and signal-to-noise ratio (SNR) of the lesion and each abdominal organ. However, this increases the beam hardening artefacts around the contrast agent-filled vessels, such as the aorta, splenic artery, or superior mesenteric artery, which may affect the evaluation of the adjacent pancreas. These limitations were compensated by the high-tube current modulation technique and IR $[16,17]$. Thus, feasible CT imaging in the adult abdominal region using low tube voltage and IR has been gradually reported. 
The reduction in the amount of iodine contrast material is another major concern in CT image acquisition because contrast material-induced nephrotoxicity is closely related to pre-existing renal insufficiency and the total amount of contrast materials [18]. We hypothesized that a higher iodine contrast enhancement increased by low tube voltage imaging can be adjusted to a level that does not affect the image quality and diagnosis by reducing the total amount of contrast medium. Therefore, the purpose of this study was to evaluate the image quality and reduce the radiation dose in pancreatic dynamic CT (PDCT) with 90kVp tube voltage and reduction of the standard amount of contrast agent based on the body weight-based adjustment table, compared with $100 \mathrm{kVp}$ PDCT and standard amount of contrast agent.

\section{Results}

1. Patient demographics and radiation dose

Fifty-one patients (mean age $65.9 \mathrm{yrs}$ ) were enrolled. The patient demographics and reduction of the radiation dose and the contrast agent volume are summarized in Table 2 . The average scan interval was 204 days ( $S D=128$ days). The mean body mass index (BMI) was 24.6 (SD, 3.1). The contrast agent used in the reduction protocol group decreased by $24.4 \%$ compared to the standard protocol group. The mean portal phase sizespecific dose estimate applying the patient's transverse and anteroposterior diameters of the case group $(9.5 \pm 2.3 \mathrm{mGy} \times \mathrm{cm})$ showed a $31.7 \%$ reduction than that of the standard protocol group $(13.9 \pm 4.8 \mathrm{mGy} \times \mathrm{cm})(p<0.001)$. The mean tube current of the portal phase in the reduction protocol group ( $245.9 \pm 69.5 \mathrm{mAs}$ ) increased by $41 \%$ compared to that in the standard protocol group $(174.2 \pm 64.0 \mathrm{mAs})(p<0.001)$ (Figure 2).

\section{Objective image analysis}

The SNR and CNR of each abdominal organ and the image noise are summarized in Table 3 , and the CNR is shown in Figure 3. Image noise of the reduction protocol group (5.4 \pm 0.9$)$ was significantly reduced compared to that of the standard protocol group $(6.8 \pm 2.4)$ by $20.6 \%(p<0.001)$. The SNR of the psoas muscle showed no significant difference between the groups $(p=0.135)$. The SNR and CNR of the aorta, liver, MPV, pancreas, spleen, and kidney were significantly higher in the reduction protocol group ( $p$ $<0.001)$.

\section{Subjective image analysis}

Reviewer 1 and reviewer 2 were off-site and on-site institution reviewers, respectively. Intraobserver and interobserver agreements were moderate to substantial $(k=0.4-0.8)$ (Table 4). The subjective image scores of both reviewers are summarized in Table 5. Except for lesion conspicuity, both reviewers judged that the image quality in the reduction protocol group was better than that in the standard protocol group. For lesion conspicuity, 40 hepatic cysts $(8 \pm 3 \mathrm{~mm}), 30$ pancreatic cystic lesions $(12 \pm 7 \mathrm{~mm})$, and five hepatic hemangiomas $(15 \pm 8 \mathrm{~mm}$ ) were evaluated. All lesions were detected by both reviewers on both groups. Lesion conspicuity was measured to be higher than 4.0 in both groups, but there was no statistically significant difference. 


\section{Discussion}

In this study, the mean amount of contrast agent in the $90 \mathrm{kVp}$ tube voltage group was reduced by $24.4 \%$ compared to the standard amount in the $100 \mathrm{kVp}$ tube voltage group. In addition to reducing the total amount of the contrast agent, the $90 \mathrm{kVp}$ tube voltage group achieved a reduction of sizespecific dose estimate by $31.7 \%$ compared with the $100 \mathrm{kVp}$ tube voltage group. Despite the decrease in the radiation dose and the amount of total contrast agent, the objective image quality was significantly better in the $90 \mathrm{kVp}$ tube voltage group. The objective image quality results were also validated by subjective image quality analysis. Each subjective image quality score was significantly higher in the $90 \mathrm{kVp}$ tube voltage scan images than $100 \mathrm{kVp}$ tube voltage scan images, except for the lesion conspicuity score. Subjective image quality was highly scored not only by the on-site institution reviewer but also by the offsite institution reviewer, who was not familiar with on-site institution images. The detection of low-density lesions in the liver and pancreas may be limited owing to the deterioration of the image quality in lowtube-voltage images. The lesion conspicuity score showed no significant difference between the case and standard protocol groups; it was measured at an average of 4 points or more, so there will be less impact on lesion detection. Based on the effect of improving the SNR and CNR of the image and the reduction of image noise and artefacts, the overall diagnostic confidence was higher in the reduction protocol group than in the standard protocol group.

The kedge of iodine at $90 \mathrm{keV}$ is higher than that at $100 \mathrm{keV}$. Therefore, theoretically, similar image quality can be obtained even with a relatively small amount of contrast agent. Buls et al. reported that when 80$k V p$ tube voltage scan was applied, a similar contrast enhancement effect could be achieved even with $30 \%$ less contrast agent compared to $120 \mathrm{kVp}$ tube voltage scan for abdominopelvic CT scan [19]. To minimize the degradation of image quality, such as unexpected deterioration of the contrast enhancement effect, we performed PDCT with $90 \mathrm{kVp}$ tube voltage and contrast medium reduced by $30 \%$ than the standard amount because CT scans in humans are greatly affected by BMI and body habitus. This study proved that a $90 \mathrm{kVp}$ low-voltage scan simultaneously reduced the radiation dose and the total amount of contrast agent without deterioration of the image quality compared to the $100 \mathrm{kVp}$ tube voltage scan.

The reduction in the radiation dose was mainly dependent on the tube potential. The radiation dose is proportional to the square of the tube potential [3]. Reducing the tube voltage from 100 to $80 \mathrm{kVp}$ reduces the radiation dose by a factor of 1.5 [20]. In a previous study, we achieved a $13.3 \%$ radiation dose reduction using $100 \mathrm{kVp}$ tube voltage with IR compared with $120 \mathrm{kVp}$ tube voltage in abdomen-pelvic CT [21]. As the tube current is directly proportional to the radiation dose, a $50 \%$ reduction in tube current results in a 50\% reduction in radiation dose. The decrease in image quality owing to a decrease in the Xray tube voltage is compensated by an increase in the tube current. In fact, in this study, as the tube voltage decreased by $10 \mathrm{kVp}$, the total radiation dose finally decreased by $31.7 \%$, although the tube current increased by $41.2 \%$. 
Marin et al. reported radiation dose reduction and improved imaging features related to contrast enhancement effects, such as CNR of the aorta, pancreas, portal vein, and pancreas to tumor and lesion conspicuity [6]. Even though we reduced the contrast medium dose to approximately $30 \%$ of the standard amount based on patient body weight, the image quality was significantly improved compared to the standard protocol group. This difference may have resulted because although the equipment used as the standard protocol group in this study had similar specifications compared to the reduction protocol group, the reduction protocol group's equipment was updated, and the latest versions of IR and high tube current techniques were installed. The reduction protocol group showed a higher mean tube current dose and lower image noise than the standard protocol group. Low tube voltage CT imaging has the disadvantage of increasing image noise, but the overall image quality is improved by removing the image noise through the IR algorithm instead of the classical image reconstruction algorithm known as filtered back-projection [22]. The IR applied in the reduction protocol group was a more advanced algorithm than that in the standard protocol group, and as a result, the image noise of the reduction protocol group was significantly lower than that of the standard protocol group by $20.6 \%$. With compensation by high tube current modulation and the latest IR technique, a $90 \mathrm{kVp}$ tube voltage scan improved both objective and subjective image quality without impairing lesion detection and diagnostic confidence despite the reduction in tube voltage and the total amount of contrast agent.

This study has several limitations. First, only a few patients with a BMI of 30 or higher were included to minimize the image quality degradation by BMI. When scanning using a $90 \mathrm{kVp}$ tube voltage was not possible, the equipment software was set to increase the tube voltage for scanning. However, this limitation also suggests that low-tube voltage imaging could be possible without deterioration of image quality in patients with a BMI less than 30 . Second, there was a difference in the equipment used between both groups. The CT used in the reduction protocol group was introduced one generation later than that of the standard protocol equipment; thus, the IR algorithm and X-ray tube technology were more advanced. Third, high-contrast lesions of the pancreas and liver, such as hepatic hemangioma, hepatocellular carcinoma, and neuroendocrine tumors of the pancreas, could not be evaluated because there were few or no lesions in the study population. However, considering the SNR and CNR of the vessels in the arterial and portal phases, these lesions have high lesion conspicuity scores.

In conclusion, the combination of $90 \mathrm{kVp}$ tube voltage, IR, and high tube current achieved a reduction of the total amount of contrast medium by $25 \%$ and the radiation dose by $31.7 \%$ without deterioration of image quality and diagnostic reliability compared with $100 \mathrm{kVp}$ tube voltage with standard amount of contrast agent in PDCT.

\section{Materials And Methods}

This retrospective study was conducted in accordance with the Helsinki declaration and approved by the Institutional Review Board and Ethics Committee of the Hallym University Sacred Heart Hospital (IRB number: 2021-08-024-001). The requirement for written informed consent was waived and confirmed by the Institutional Review Board and Ethics Committee of the Hallym University Sacred Heart Hospital. This 
study design followed the Strengthening the Reporting of Observational Studies in Epidemiology (STROBE) guidelines.

\section{Sample size estimation and patient enrollment}

This study was designed for matched pairwise comparison. The minimum sample size was determined to 44 patients under the assumption of effect size of 0.5 , a priory statistical significance of 0.05 and a power of $90 \%$.

From July 2020 to June 2021, 371 patients received 90kVp tube voltage and reduced contrast agent administration protocol. Among them, 57 patients above 18 years of age received both $90 \mathrm{kVp}$ and 100 $k V p$ tube voltage PDCT within the same period. Six patients were excluded because of severe motion artifacts $(n=1)$, severe generalized edema-related pancreatitis or recent pancreatic surgery $(n=3)$, and no information on body mass index $(n=4)$ (Figure 1). Ultimately, 51 patients were enrolled in the study.

\section{CT protocol}

All CT images were obtained using one of three machines. The reduction protocol group was scanned with $90 \mathrm{kVp}$ tube voltage and $374 \mathrm{mAs}$ or $393 \mathrm{mAs}$ of reference tube current using MDCT scanners (SOMATOM Force, Siemens Healthineers) with a reduction of the standard amount of contrast material (300 mg of iodine per $\mathrm{mL}$, approximately $1.4 \mathrm{ml} / \mathrm{kg}$, according to the dosage adjustment table (Table 1) and reconstructed by advanced IR algorithm (ADMIRE strength 2, Br40 of the reconstruction kernel). The standard protocol group was scanned with a $100 \mathrm{kVp}$ tube voltage and $289 \mathrm{mAs}$ of reference tube current using two MDCT scanners (SOMATOM Definition Edge or SOMATOM Definition Flash; Siemens Healthineers) using a standard amount of contrast material (300 mg of iodine per $\mathrm{mL}, 2 \mathrm{ml} / \mathrm{kg}$ ) and reconstructed using the IR algorithm (SAFIRE strength 2, 140 of reconstruction kernel). PDCT consists of a dual phase (arterial and portal venous phases). All patients received an intravenous nonionic contrast medium containing an iodine concentration of $300 \mathrm{mg} / \mathrm{mL}$ (Bonorex 300 [iohexol], Central Medical Service, Korea; lomerolâ 300 [iomeprol], Bracco Imaging, Korea). For dynamic imaging, nonionic contrast material per kilogram of body weight was administered at a rate of $25 \mathrm{~mL} / \mathrm{s}$ using an automatic power injector (Multilevel CT, Medrad, USA), followed by a $20 \mathrm{~mL}$ flush of sterile saline. Bolus tracking method was used to determine the timing of arterial phase scanning, and the arterial phase was obtained 15 seconds after triggering when the proximal abdominal aorta reached the Hounsfield unit of 100 or greater. Portal venous phase images were acquired 30 seconds after the end of arterial phase scan. The reconstruction parameters of the axial image were a $3 \mathrm{~mm}$ section thickness and a $3 \mathrm{~mm}$ reconstruction interval. A coronal reformatted image was reconstructed with a section thickness of $3 \mathrm{~mm}$ and an interval of $3 \mathrm{~mm}$. Table 2 is comparison of both CT acquisition protocols.

\section{Radiation dose evaluation}

The radiation dose of the pre-contrast images was excluded from the evaluation because the $k \mathrm{p}$ of the pre-contrast scan was not fixed. The only radiation dose in the portal phase images was compared. Size- 
specific dose estimation was calculated using the sum of the anteroposterior and transverse dimensions according to the report 204 released by the American Association of Physicists in Medicine (AAPM) [23]. The effective tube current (mAs) of each patient in the portal phase was recorded.

\section{Image analysis}

\subsection{Objective assessment of image quality}

The standard deviation (SD) of the air located outside the patient's xyphoid process level was defined as the objective image noise. The mean CT Hounsfield units (HU) of each abdominal organ, such as the aorta, liver, main portal vein (MPV), pancreas, spleen, kidney, and psoas muscle, as well as cystic lesions of the pancreas and liver which is more than $5 \mathrm{~mm}$ in diameter, were measured using a circular region of interest. When drawing ROls in each target organ, special care was taken not to include adjacent vessels, bile ducts, artefacts, or peritoneal fat.

The mean CT HU of the aorta, pancreatic parenchyma, and psoas muscle were measured in both the arterial and portal phases. The mean CT HU values of the liver, MPV, spleen, and kidney were measured only during the portal phase of CT scanning. Liver attenuation was recorded as the mean of the measurements of four ROIs in the medial and lateral segments of the left hepatic lobe, and the anterior and posterior segments of the right hepatic lobe [24]. Aortal attenuation was measured at the celiac trunk take-off level. The attenuation of the psoas muscle was recorded as the mean attenuation of two ROls that avoided macroscopic fat infiltration at the L4 vertebral level. Kidney attenuation was measured in the renal cortex, with special care taken to avoid containing the medulla and perirenal fat. The size, shape, and position of all ROI measurements were kept constant by applying a copy-and-paste function at the workstation. The SNR and CNR were calculated as follows:

$\mathrm{SNR}_{\text {target organ }}=\mathrm{HU}_{\text {target organ }} /$ background image noise

$\mathrm{CNR}_{\text {target organ }}=\left(\mathrm{HU}_{\text {target organ }}-\mathrm{HU}_{\text {psoas muscle }}\right) /$ background image noise

\subsection{Subjective image quality analysis}

The subjective image quality analysis was independently and blindly evaluated by two board-certified radiologists with more than 10 years of experience. One reviewer was from an off-site institution. Subjective image noise, beam hardening or streak artefacts, visibility of small structures (peripheral hepatic vessels), lesion conspicuity, and overall diagnostic confidence were evaluated using a 5point scale based on the European Guidelines on Quality Criteria for Computed Tomography and previous research published in the radiology literature [7, 24-26]. All 102 PDCT sets were reviewed by two reviewers without any information of patient and scan technique. Subjective image noise was graded on a 5 point scale based on the presence and amount of image mottle or graininess (5, minimal image noise; 4 , less than average noise; 3 , average image noise; 2 , above average noise; 1 , unacceptable image noise). The visibility of small structures, mainly hepatic vessels, was also graded using a 5-point scale, with 5 
indicating excellent visualization and 1 indicating imperceptible small hepatic vessel structures. Beam hardening or streak artefacts were graded on a 5 point scale (5, absence of artefact; 4 , mild artefacts not interfering with diagnosis; 3 , moderate artefacts slightly interfering with diagnosis; 2 , pronounced artefacts interfering with diagnosis; and 1, impossible interpretation of a lesion or an organ of interest).

Hepatic cysts, pancreatic cystic lesions and hepatic hemangiomas more than $5 \mathrm{~mm}$ in short diameter were selected by an independent researcher for lesion conspicuity evaluation. Each reviewer received brief lesion information including image number on arterial or portal phase, anatomic location or adjacent anatomic landmark.

Lesion conspicuity was ranked on a 5 point scale with a score of 5 indicating a clearly seen lesion with clearly visualized margins and a score of 1 indicating an imperceptible lesion. Lesion conspicuity was evaluated based on the visibility of the lesion boundary. When more than $75 \%$ of the boundary of the lesion was visible, it was marked as 5 points; when $50 \% 75 \%$ of the boundary was visible, it is marked as 4 points; when $2550 \%$ of the boundary was visible, it is marked as 3 points; and when only less than $25 \%$ of the boundary was visible, it was marked as 2 . Overall diagnostic confidence was evaluated using a 5 point scale; grade 1, nonconfident; grade 2 , subdiagnostic confidence; grade 3 , average confidence; grade 4 , more than average; and grade 5 , completely confident.

\section{Statistics}

Continuous variables are expressed as means and SD. Intraobserver and interobserver agreements were assessed using the weighted kappa statistics. Quantitative image parameters (attenuation values, image noise, SNR, and CNR) were corrected using Welch's test depending on normality testing and compared using paired t-tests. Qualitative subjective image analysis of the image was performed using the Wilcoxon signed-rank test. Statistical analysis was performed using the MedCalc software (MedCalc 13.1.2). Statistical significance was set at $p<0.05$.

\section{Data Availability}

The dataset used in this study is not publicly available due to patient privacy constraints and data availability policy of our institution committee. An anonymized version of this dataset can be available from the corresponding author on reasonable request.

\section{Abbreviation}

IR, iterative reconstruction PDCT, pancreas dynamic CT

\section{Declarations}

\section{Acknowledgements}

Nothing to state 


\section{Author Contribution statements}

Conceptualization: Hong II Ha

Data curation: Min Su Park, Hong II Ha, Jhii-Hyun Ahn

Formal analysis: Min Su Park, Hong II Ha, Jhii-Hyun Ahn

Funding acquisition: N/A

Investigation: Min Su Park, Hong II Ha

Methodology: Hong II Ha, Jhii-Hyun Ahn, Sang Min Lee

Project administration: Hong II Ha

Resources: Hong II Ha

Software: Hong II Ha

Supervision: Hong II Ha, In Jae Lee

Validation: Hong II Ha, In Jae Lee

Visualization: Min Su Park, Hong II Ha

Writing-original draft: Min Su Park, Hong II H

Writing-review \& editing: Hong II Ha, Jhii-Hyun Ahn, Sang Min Lee

\section{Competing interests}

All authors have nothing to declare.

\section{References}

1. Mettler, F. A. Jr. et al. Radiologic and nuclear medicine studies in the United States and worldwide: frequency, radiation dose, and comparison with other radiation sources-1950-2007. Radiology 253, 520-531 (2009).

2. Goo, H. W. CT radiation dose optimization and estimation: an update for radiologists. Korean J. Radiol. 13, 1-11 (2012).

3. Kalra, M. K. et al. Strategies for CT radiation dose optimization. Radiology 230, 619-628 (2004)

4. Kalender, W. A. et al. Technical approaches to the optimisation of CT. Phys. Med. 24, 71-79 (2008).

5. Winklehner, A. et al. Raw data-based iterative reconstruction in body CTA: evaluation of radiation dose saving potential. Eur. Radiol. 21, 2521-2526 (2011). 
6. Marin, D. et al. Low-tube-voltage, high-tube-current multidetector abdominal CT: improved image quality and decreased radiation dose with adaptive statistical iterative reconstruction algorithminitial clinical experience. Radiology 254, 145-153 (2010).

7. Hur, S. et al. 80-kVp CT using Iterative Reconstruction in Image Space algorithm for the detection of hypervascular hepatocellular carcinoma: phantom and initial clinical experience. Korean J. Radiol. 13, 152-164 (2012).

8. den Harder, A. M. et al. Hybrid and model-based iterative reconstruction techniques for pediatric CT. Am. J. Roentgenol. 204, 645-653 (2015).

9. Lee, K. B. \& Goo, H. W. Quantitative Image Quality and Histogram-Based Evaluations of an Iterative Reconstruction Algorithm at Low-to-Ultralow Radiation Dose Levels: A Phantom Study in Chest CT. Korean J. Radiol. 19, 119-129 (2018).

10. Nagayama, Y. et al. Radiation Dose Reduction at Pediatric CT: Use of Low Tube Voltage and Iterative Reconstruction. Radiographics 38, 1421-1440 (2018).

11. Pontana, F. et al. Reduced-dose low-voltage chest CT angiography with Sinogram-affirmed iterative reconstruction versus standard-dose filtered back projection. Radiology 267, 609-618 (2013).

12. Nakayama, Y. et al. Abdominal CT with low tube voltage: preliminary observations about radiation dose, contrast enhancement, image quality, and noise. Radiology 237, 945-951 (2005).

13. Seyal, A. R. et al. CT of the Abdomen with Reduced Tube Voltage in Adults: A Practical Approach. Radiographics 35, 1922-1939 (2015).

14. Dong, F., Davros, W., Pozzuto, J. \& Reid, J. Optimization of kilovoltage and tube current-exposure time product based on abdominal circumference: an oval phantom study for pediatric abdominal CT. Am. J. Roentgenol. 199, 670-676 (2012).

15. Kim, S. Y. et al. Low-Tube-Voltage CT Urography Using Low-Concentration-lodine Contrast Media and Iterative Reconstruction: A Multi-Institutional Randomized Controlled Trial for Comparison with Conventional CT Urography. Korean J. Radiol. 19, 1119-1129 (2018).

16. Kaza, R. K. et al. Emerging techniques for dose optimization in abdominal CT. Radiographics 34, 417 (2014).

17. Shin, H. J. et al. Radiation dose reduction via sinogram affirmed iterative reconstruction and automatic tube voltage modulation (CARE kV) in abdominal CT. Korean J. Radiol. 14, 886-893 (2013).

18. Davenport, M. S. et al. Contrast material-induced nephrotoxicity and intravenous low-osmolality iodinated contrast material. Radiology 267, 94-105 (2013).

19. Brady, S. L. \& Kaufman, R. A. Investigation of American Association of Physicists in Medicine Report 204 size-specific dose estimates for pediatric CT implementation. Radiology 265, 832-840 (2012).

20. Singh, S. et al. Dose reduction and compliance with pediatric CT protocols adapted to patient size, clinical indication, and number of prior studies. Radiology 252, 200-208 (2009). 
21. Panzer, W., Shrimpton, P. C. \& Jessen, K. European guidelines on quality criteria for computed tomography. EU publications https://op.europa.eu/en/publication-detail/-/publication/d229c9e1a967-49de-b169-59ee68605f1a (2000).

22. Song, J. S., Choi, E. J., Kim, E. Y., Kwak, H. S. \& Han, Y. M. Attenuation-based automatic kilovoltage selection and sinogram-affirmed iterative reconstruction: effects on radiation exposure and image quality of portal-phase liver CT. Korean J. Radiol. 16, 69-79 (2015).

23. Buls, N. et al. Contrast agent and radiation dose reduction in abdominal CT by a combination of low tube voltage and advanced image reconstruction algorithms. Eur. Radiol. 25, 1023-1031 (2015).

24. Karmazyn, B., Liang, Y., Klahr, P. \& Jennings, S. G. Effect of tube voltage on CT noise levels in different phantom sizes. Am. J. Roentgenol. 200, 1001-1005 (2013).

25. Ha, H. I., Hong, S. S., Kim, M. J. \& Lee, K. 100 kVp Low-Tube Voltage Abdominal CT in Adults: Radiation Dose Reduction and Image Quality Comparison of $120 \mathrm{kVp}$ Abdominal CT. J. Korean Soc. Radiol. 75, 285-295 (2016).

26. Padole, A., Ali Khawaja, R. D., Kalra, M. K. \& Singh, S. CT radiation dose and iterative reconstruction techniques. Am. J. Roentgenol. 204, 384-392 (2015).

\section{Tables}

Table 1. The contrast agent administration adjustment table of the $90-\mathrm{kVp}$ pancreas dynamic CT protocol. 


\begin{tabular}{|lll|}
\hline Body weight $(\mathrm{kg})$ & Contrast agent volume $(\mathrm{ml})$ & Flow rate $(\mathrm{ml} / \mathrm{sec})$ \\
\hline 40 & 60 & 2.0 \\
\hline 45 & 68 & 2.0 \\
\hline 50 & 75 & 2.0 \\
\hline 55 & 83 & 2.1 \\
\hline 60 & 90 & 2.3 \\
\hline 65 & 98 & 2.4 \\
\hline 70 & 105 & 2.6 \\
\hline 75 & 113 & 2.8 \\
\hline 80 & 120 & 3.0 \\
\hline 85 & 128 & 3.2 \\
\hline 90 & 135 & 3.4 \\
\hline 95 & 143 & 3.6 \\
\hline 100 & 150 & 3.8 \\
\hline 105 & 158 & 3.9 \\
\hline 110 & 165 & 4.1 \\
\hline 115 & 173 & 4.3 \\
\hline 120 & 180 & 4.5 \\
\hline
\end{tabular}

Table 2. Patient demographics, and comparison of CT scan protocol, radiation dose and contrast agent volume reduction. 


\begin{tabular}{|c|c|c|c|}
\hline & $\begin{array}{l}\text { Reduction protocol } \\
\text { group } \\
\text { (90-kVp tube voltage } \\
\& \\
\text { reduced contrast } \\
\text { agent volume) }\end{array}$ & $\begin{array}{l}\text { Standard protocol group } \\
\text { (100-kVp tube voltage \& standard dose } \\
\text { of contrast agent) }\end{array}$ & $\begin{array}{l}p- \\
\text { value }\end{array}$ \\
\hline Age (yrs.) & $65.9 \pm 9.4$ & & - \\
\hline Sex (M:F) & $23 \pm 28$ & & - \\
\hline Height (cm) & $159.5 \pm 9.4$ & & - \\
\hline Weight (kg) & $62.9 \pm 11.2$ & & - \\
\hline $\mathrm{BMI}\left(\mathrm{m}^{2} / \mathrm{kg}\right)$ & $24.6 \pm 3.1$ & & - \\
\hline CT interval (days) & $203.8 \pm 128.4$ & & - \\
\hline CT scanner & Somatom Force & Somatom Definition Edge or Flash & - \\
\hline kVp & $90-k V p$ & 100-kVp & - \\
\hline Reference tube current & $289 \mathrm{mAs}$ & 374-393 mAs & $\begin{array}{l}< \\
0.001\end{array}$ \\
\hline $\begin{array}{l}\text { Effective tube current } \\
\text { (mAs) }\end{array}$ & $245.9 \pm 69.5$ & $174.2 \pm 64.0$ & $\begin{array}{l}<.001 \\
0.001\end{array}$ \\
\hline $\begin{array}{l}\text { Contrast material volume } \\
(\mathrm{ml})\end{array}$ & $95.5 \pm 17.0$ & $126.3 \pm 22.3$ & $\begin{array}{l}< \\
0.001\end{array}$ \\
\hline $\begin{array}{l}\text { Sizespecific dose } \\
\text { estimate }(\mathrm{mGy} \times \mathrm{cm})\end{array}$ & $9.5 \pm 2.3$ & $13.9 \pm 4.8$ & $\begin{array}{l}<.001 \\
0.001\end{array}$ \\
\hline $\begin{array}{l}\text { Iteration technique \& } \\
\text { strength }\end{array}$ & SAFIRE, strength 2 & ADMIRE, strength 2 & - \\
\hline Reconstruction kernel & 140 & $\mathrm{Br} 40$ & - \\
\hline Slice thickness \& interval & $3 \mathrm{~mm} \& 3 \mathrm{~mm}$ & $3 \mathrm{~mm} \& 3 \mathrm{~mm}$ & - \\
\hline
\end{tabular}

Table 3. Paired $t$ test results of objective image quality. 


\begin{tabular}{|c|c|c|c|}
\hline & $\begin{array}{l}\text { Reduction protocol } \\
\text { aroup }\end{array}$ & \multirow{3}{*}{$\begin{array}{l}\text { Standard protocol group } \\
\text { (100-kVp tube voltage \& standard dose } \\
\text { of contrast agent) }\end{array}$} & \multirow[t]{3}{*}{$p$-value } \\
\hline & $\begin{array}{l}(90-k V p \text { tube voltage } \\
\&\end{array}$ & & \\
\hline & $\begin{array}{l}\text { reduced contrast } \\
\text { agent volume) }\end{array}$ & & \\
\hline $\begin{array}{l}\text { Image noise (arterial } \\
\text { phase) }\end{array}$ & $5.3 \pm 1.1$ & $6.2 \pm 1.7$ & $<0.001$ \\
\hline $\begin{array}{l}\text { Image noise (portal } \\
\text { phase) }\end{array}$ & $5.4 \pm 0.9$ & $6.8 \pm 2.4$ & $<0.001$ \\
\hline \multicolumn{4}{|l|}{ Signal to Noise Ratio } \\
\hline $\begin{array}{l}\text { Aorta (arterial } \\
\text { phase) }\end{array}$ & $81.1 \pm 23.8$ & $48.9 \pm 19.0$ & $<0.001$ \\
\hline $\begin{array}{l}\text { Aorta (portal } \\
\text { phase) }\end{array}$ & $39.7 \pm 8.7$ & $29.1 \pm 9.5$ & $<0.001$ \\
\hline Liver (portal phase) & $24.4 \pm 6.4$ & $18.6 \pm 6.7$ & $<.001$ \\
\hline $\begin{array}{l}\text { Main portal vein } \\
\text { (portal phase) }\end{array}$ & $43.0 \pm 9.0$ & $30.6 \pm 10.7$ & $<0.001$ \\
\hline $\begin{array}{l}\text { Pancreas (arterial } \\
\text { phase) }\end{array}$ & $22.9 \pm 6.0$ & $17.0 \pm 7.7$ & $<001$ \\
\hline $\begin{array}{l}\text { Pancreas (portal } \\
\text { phase) }\end{array}$ & $21.5 \pm 4.8$ & $16.3 \pm 5.6$ & $<001$ \\
\hline $\begin{array}{l}\text { Spleen (portal } \\
\text { phase) }\end{array}$ & $29.3 \pm 6.3$ & $21.5 \pm 7.4$ & $<001$ \\
\hline $\begin{array}{l}\text { Renal cortex (portal } \\
\text { phase) }\end{array}$ & $45.1 \pm 9.8$ & $33.7 \pm 12.2$ & $<0.001$ \\
\hline $\begin{array}{l}\text { Psoas muscle } \\
\text { (arterial phase) }\end{array}$ & $11.7 \pm 2.8$ & $10.8 \pm 2.6$ & 0.213 \\
\hline $\begin{array}{l}\text { Psoas muscle } \\
\text { (portal phase) }\end{array}$ & $11.4 \pm 3.2$ & $10.4 \pm 3.2$ & 0.135 \\
\hline \multicolumn{4}{|l|}{$\begin{array}{l}\text { Contrast to Noise } \\
\text { Ratio }\end{array}$} \\
\hline $\begin{array}{l}\text { Aorta (arterial } \\
\text { phase) }\end{array}$ & $59.3 \pm 15.1$ & $40.9 \pm 18.8$ & $<0.001$ \\
\hline $\begin{array}{l}\text { Aorta (portal } \\
\text { phase) }\end{array}$ & $27.4 \pm 6.9$ & $19.6 \pm 7.0$ & $<001$ \\
\hline Liver (portal phase) & $12.1 \pm 4.8$ & $9.0 \pm 4.1$ & 0.001 \\
\hline
\end{tabular}


Main portal vein (portal phase)

$30.6 \pm 7.2$

$11.2 \pm 4.4$ phase)

Pancreas (arterial

Pancreas (portal phase)

Spleen (portal phase)

Renal cortex (portal $\quad 32.8 \pm 8.0$ phase)

Table 4. Intra-rater and inter-rater agreement of subjective image quality score.

\begin{tabular}{|lll|}
\hline & Intra-rater agreement (ICC) & Inter-rater agreement (kappa) \\
\hline Subjective image noise & $0.7(0.6-0.8)$ & $0.5(0.4-0.6)$ \\
\hline $\begin{array}{l}\text { Visibility of small structures } \\
\text { Visibility of peripheral hepatic vessels) }\end{array}$ & $0.8(0.7-0.8)$ & $0.5(0.4-0.7)$ \\
\hline Beam hardening or streak artifact & $0.5(0.4-0.7)$ & $0.4(0.3-0.6)$ \\
\hline Lesion conspicuity & $0.8(0.7-0.8)$ & $0.4(0.3-0.5)$ \\
\hline Overall diagnosis confidence & $0.7(0.5-0.8)$ & $0.5(0.3-0.7)$ \\
\hline
\end{tabular}

Numbers in parentheses are $95 \%$ confidence interval.

Table 5. Paired comparison of subjective image quality by the Wilcoxon signed-rank test. 


\begin{tabular}{|c|c|c|c|c|c|c|}
\hline & \multicolumn{3}{|l|}{ Reviewer 1} & \multicolumn{3}{|l|}{ Reviewer 2} \\
\hline & & $\begin{array}{l}\text { Standard } \\
\text { protocol group }\end{array}$ & $\begin{array}{l}p- \\
\text { value }\end{array}$ & Reduction & $\begin{array}{l}\text { Standard } \\
\text { protocol group }\end{array}$ & $\begin{array}{l}p- \\
\text { value }\end{array}$ \\
\hline & $\begin{array}{l}\text { protocol } \\
\text { group }\end{array}$ & & & $\begin{array}{l}\text { protocol } \\
\text { group }\end{array}$ & & \\
\hline $\begin{array}{l}\text { Subjective image } \\
\text { noise }\end{array}$ & $4.3 \pm 0.5$ & $3.4 \pm 0.7$ & $<.001$ & $3.5 \pm 0.5$ & $2.7 \pm 0.6$ & $\begin{array}{l}< \\
0.001\end{array}$ \\
\hline $\begin{array}{l}\text { Visibility of small } \\
\text { structures }\end{array}$ & $3.6 \pm 0.5$ & $3.1 \pm 0.7$ & $<.001$ & $3.6 \pm 0.5$ & $3.1 \pm 0.6$ & $\begin{array}{l}< \\
0.001\end{array}$ \\
\hline $\begin{array}{l}\text { (Visibility of } \\
\text { peripheral hepatic } \\
\text { vessels) }\end{array}$ & & & & & & \\
\hline $\begin{array}{l}\text { Beam hardening or } \\
\text { streak artifact }\end{array}$ & $4.9 \pm 0.3$ & $4.8 \pm 0.4$ & 0.086 & $4.3 \pm 0.5$ & $3.9 \pm 0.7$ & 0.002 \\
\hline Lesion conspicuity & $4.7 \pm 0.6$ & $4.8 \pm 0.4$ & 0.261 & $4.1 \pm 1.0$ & $4.0 \pm 1.1$ & 0.352 \\
\hline $\begin{array}{l}\text { Overall diagnostic } \\
\text { confidence }\end{array}$ & $4.9 \pm 0.3$ & $4.5 \pm 0.6$ & $<.001$ & $4.6 \pm 0.8$ & $3.9 \pm 0.9$ & $\begin{array}{l}<.001 \\
0.001\end{array}$ \\
\hline
\end{tabular}

\section{Figures}




\section{Between 2020.July and 2021.June}

\begin{tabular}{|c|}
\hline $\begin{array}{c}\text { Pancreas dynamic CT with reduction protocol; } \\
90-\mathrm{kVp} \& \text { reduced contrast agent volume } \\
(n=271)\end{array}$ \\
\begin{tabular}{|c|}
\hline Inclusion criteria $(n=91) ;$ \\
age $>18$ years old \\
\& underwent 100-kVp standard protocol CT \\
\& contrast agent concentration: 300 mgl/100ml
\end{tabular} \\
CT within 1-month after major pancreatic surgery $(n=23)$ \\
or severe motion artifact $(n=3)$ \\
or no body mass index information $(n=14)$ \\
\hline \\
\hline
\end{tabular}

Figure 1

Patient selection flowchart. 


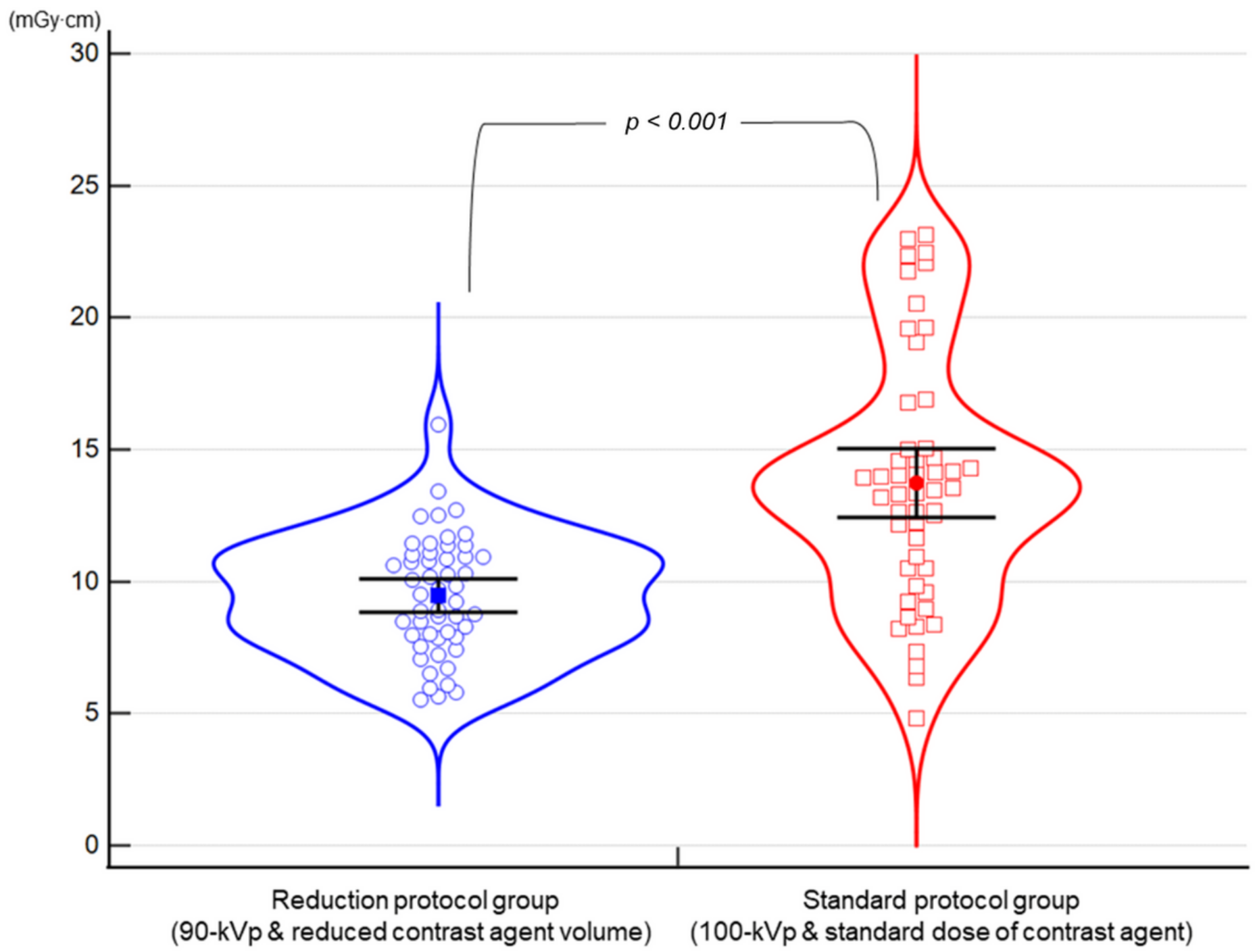

Figure 2

Violin plot comparison of size-specific dose estimation. 


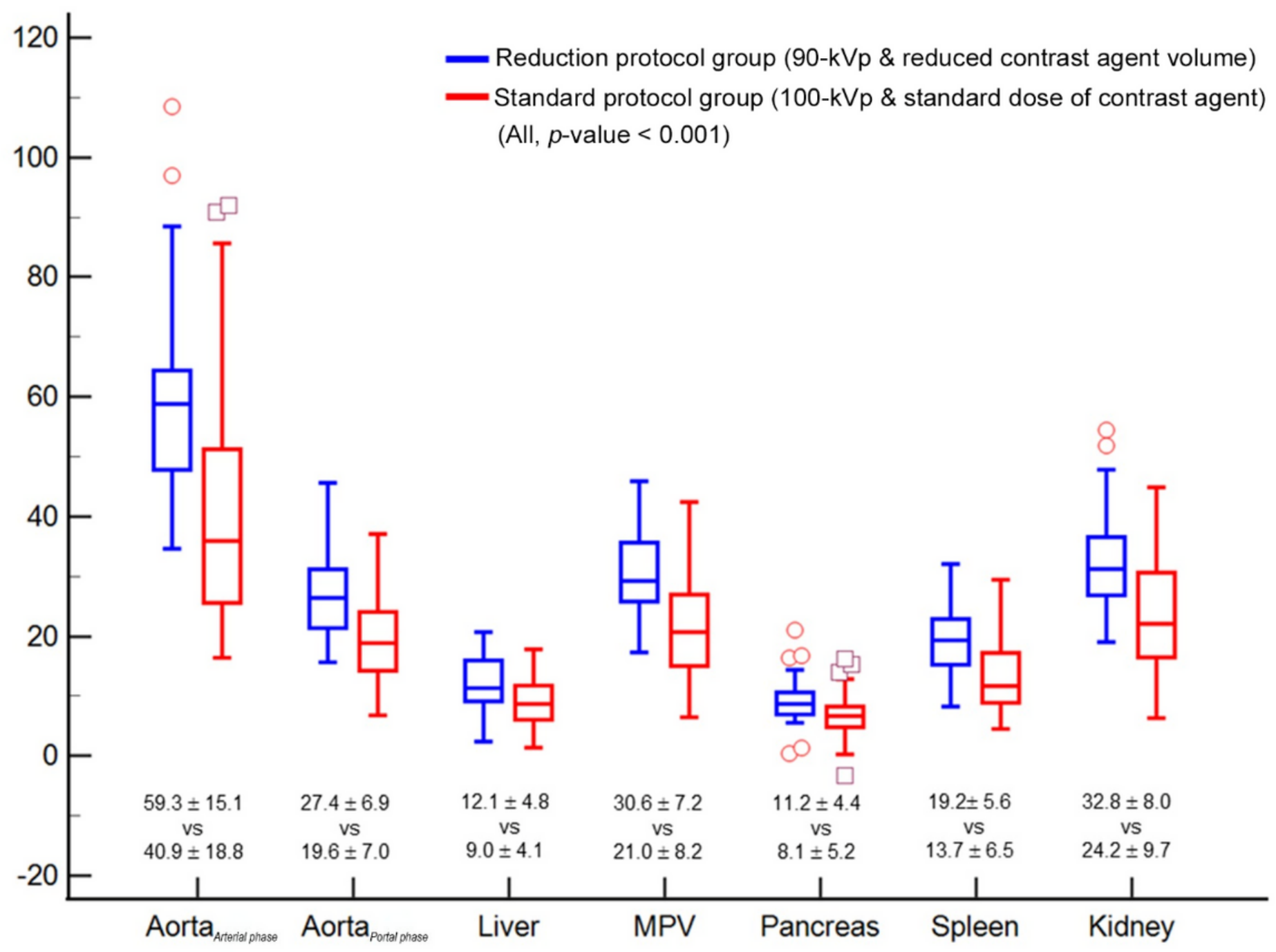

Figure 3

Box plot comparison of contrast-to-noise ratio of each abdominal organs (Aorta ${ }_{\text {Arterial phase }}$ and Aorta $_{\text {Portal }}$ phase are value measured on arterial and portal phase, respectively).

\section{Supplementary Files}

This is a list of supplementary files associated with this preprint. Click to download.

- SupplementaryFigure1S.pdf 